\title{
Consumers' evaluation of allocation policies for scarce health care services: Vested interest activation trumps spatial and temporal distance
}

\author{
Tim M. Benning • Els Breugelmans • Benedict G. C. Dellaert
}

Published online: 26 January 2012

(C) The Author(s) 2012. This article is published with open access at Springerlink.com

\begin{abstract}
The allocation of scarce health care service resources often requires tradeoffs between individual and collective outcomes (e.g., when some individuals benefit more strongly from a given policy than others). Based on construal level theory, one would expect that consumers cognitively represent the individual and collective outcomes of an allocation policy at different levels of abstraction and that they evaluate allocation policies more positively when there is congruency between the cognitive representation of the policy's focal outcome and the spatial and temporal distance inherently present in the policy's decision context (e.g., allocation decisions on a future policy). However, we hypothesize that this congruency effect can be overruled by a high vested interest mindset that is activated by an individual's recent personal experience with a health care service provider. Since a high vested interest mindset increases the relevance of the allocation policy implementation for the individual because s/he perceives strong personal consequences, we propose that for consumers with recent experience the evaluation of an allocation policy that focuses on individual outcomes is higher than that of a policy that focuses on collective outcomes irrespective of the spatial and temporal distance in the decision
\end{abstract}

T. M. Benning $(\bowtie)$

Institute of Health Policy \& Management, Institute for Medical Technology Assessment, Erasmus University Rotterdam, P.O. Box 1738, 3000 DR Rotterdam, The Netherlands e-mail: benning@bmg.eur.nl

\section{E. Breugelmans}

Department of Business Studies, Lessius University College,

Korte Nieuwstraat 33, 2000 Antwerp, Belgium

E. Breugelmans

Faculty of Business and Economics, Catholic University of Leuven,

Naamsestraat 69, 3000 Leuven, Belgium

e-mail: els.breugelmans@lessius.eu

B. G. C. Dellaert

Department of Business Economics, Marketing Section, Erasmus School of Economics, Erasmus University Rotterdam, P.O. Box 1738, 3000 DR Rotterdam, The Netherlands e-mail: dellaert@ese.eur.nl 
context. Results of a hypothetical experiment among a representative sample of the general population confirm the congruency effects in the new domain of health care service allocation policies, and provide support for the proposed overruling effect of the activation of a high vested interest mindset by recent personal experience on spatial and temporal distance.

Keywords Allocation policy evaluations · Individual vs. collective outcomes · Construal level theory · Vested interest theory $\cdot$ Consumer decision making

\section{Introduction}

The scarcity of service resources in important domains such as health, recreation, and education often demands that service providers implement allocation policies to assign service access to consumers (Kahneman et al. 1986; Persad et al. 2009). We define a service allocation policy as a set of rules used to decide on the allocation of access to a scarce service resource among potential recipients (e.g., based on price, time of arrival, or medical urgency). Allocation policies are adopted in many domains where service resources are scarce (e.g., doctors may use urgency indicators to decide on the order in which patients are treated, music or sport fans may be asked to wait in line to obtain access to tickets for special events, and aptitude test scores may be used to grant students access to educational programs). Typically, managers and allocation policy decision makers that need to decide on the type and specification of an allocation policy focus on both individual and collective outcomes because the maximization of one outcome may come at the expense of the other (Lindholm et al. 1997). For example, in health care, the use of an allocation policy based on urgency may maximize the number of life years saved for society (collective outcome), but not for each specific participant him- or herself (individual outcome).

Based on construal level theory (CLT), one would expect that consumers mentally represent these different allocation policy outcomes at different levels of abstraction (Liberman and Trope 1998; Trope and Liberman 2003). Policy outcomes that benefit society (collective outcomes) are likely to be seen as more distant and construed at a more abstract level than policy outcomes that directly benefit the individual him- or herself (individual outcomes), because others in society are seen as distal social targets whereas the self is seen as a proximal social target (Kim et al. 2008; Liviatan et al. 2008; Zhao and Xie 2011). In addition, prior research has shown that congruency between the consumer's mental representation and the construal level caused by the decision context results in greater processing fluency (Kim et al. 2009; White et al. 2011) and enhanced evaluations (Sanna et al. 2010; Zhao and Xie 2011). Using these insights, we expect that allocation policy evaluations are more positive when there is congruency between the temporal and spatial distance that is inherently present in the policy's decision context (e.g., an allocation policy implementation in the near future or close vicinity vs. distant future or far vicinity; Milkman et al. 2010; Soman et al. 2005) and a consumer's mental representation of the policy's focal outcome.

The main contribution of this study is to investigate the potential overruling effect of the activation of a high vested interest mindset on this CLT-based congruency 
effect. Contexts that are of very high relevance to the consumer and where perceived personal stakes are large can potentially activate a high vested interest mindset (Thornton and Knox 2002). Often, the implementation of an allocation policy where individual and collective outcomes are traded off is such a context. The implementation of health care service allocation policies, where such individual vs. collective outcome trade-offs are prominent, is also an interesting and important domain to investigate whether CLT-based congruency effects hold. This constitutes the second contribution of this study.

We propose that a high vested interest mindset can be activated by a consumer's recent personal experience with the service resource, because consumers with recent experience are better able to assess the pros and cons of a particular behavior (there is more at stake), are more aware of the effects of an action upon oneself (the action is more salient), and can better assess the chances that a specific event will take place (the results are more certain) (Crano 1995; Morwitz 1997). Vested interest theory predicates that participants with a high vested interest mindset have well-established and consistent preferences for personal (i.e., individual) consequences (e.g., Lehman and Crano 2002). Consequently, we expect them to expend more cognitive effort and produce issue-relevant thoughts (Crano 1995; Crano and Prislin 1995; Lehman and Crano 2002) and to be more likely to activate concrete, egocentric ("not in my backyard") thoughts that overrule the CLT-based congruency effect. We hypothesize that this results in a more positive evaluation of an allocation policy that focuses on individual rather than collective outcomes, regardless of the temporal or spatial distance. To the best of our knowledge, we are the first to show that there can be boundaries of an internal mindset to the previously found CLT-based congruency effect.

\section{Allocation policy evaluations}

\subsection{Construal level of individual vs. collective allocation outcomes}

Management decisions on how to allocate scarce service resources may create conflicts between collective interests and interests of different individuals. Not only managers and policy makers typically trade off these interests when deciding on the mechanism for an allocation policy, but also the recipients of the allocation policy (i.e., consumers) actively take into account the interests of others, along with their own selfinterest, when evaluating allocation policies (Agerström and Björklund 2009a, b). Such a context where perceived personal consequences for the daily life of consumers are significant and important (i.e., high hedonic relevancy and high perceived stake) can be considered as a context where a high vested interest mindset can potentially be activated (Crano 1995; Thornton and Knox 2002). Little is known about how consumers evaluate allocation policies in such a decision context, and-using construal level theory as a base-we anticipate that it depends on their mental representation of the focal outcome presented in the policy. CLT posits that individuals focus on more concrete, low-level construals when the psychological distance is close whereas focus is placed on more abstract, high-level construals when the psychological distance is far (Kardes et al. 2006; Liberman et al. 2007; Trope and Liberman 
2003). Low-level construals represent information that is concrete and subordinate, and highlight the 'how' aspects of an object/action, while high-level construals represent abstract and superordinate information and highlight the 'why' aspects of an object/action (Trope and Liberman 2003; Trope et al. 2007). Although CLT originated with the temporal distance perspective (near vs. distant future; Liberman and Trope 1998), it has been extended to other dimensions such as spatial, hypothetical, or social distance as well (Trope and Liberman 2010; Trope et al. 2007). The latter (social distance) is especially relevant in the context of selfish hedonistic (individual) vs. altruistic moral (collective) allocation policy outcomes, where prior research has shown that distal social targets (e.g., others) are represented on a more abstract level than proximal social targets (e.g., self) (Kim et al. 2008; Liviatan et al. 2008; Zhao and Xie 2011). As a consequence, we expect that collective (more altruistic) outcomes are construed at a more abstract level than individual (more selfish) outcomes when consumers evaluate an allocation policy.

\subsection{Congruency of allocation outcome construal, and spatial and temporal distance}

Consumers' allocation policy evaluations depend on congruency between (1) the construal of the allocation policy's focal outcome and (2) the spatial and temporal distance that is inherently connected to the decision context (e.g., a decision that is implemented for the distant vs. near future, or for a place in the far vs. close vicinity). A mental representation match (congruency) between the decision context and the information presented on the focal object has positive effects on the evaluation of the object (Kim et al. 2009; Sanna et al. 2010; Zhao and Xie 2011). This positive congruency effect can be explained by the value of fit (Zhao and Xie 2011) which increases processing fluency and ease of comprehension (Kim et al. 2009; Novemsky et al. 2007; White et al. 2011) and positively affects evaluation outcomes like attitude and preference (Sanna et al. 2010). Kim et al. (2009) show that responses to a concrete (low-level) vs. abstract (high-level) campaign of a fictional political candidate are more favored when respondents are told that the campaign would launch 1 week from now (near future) vs. 6 months from now (distant future), respectively. Zhao and Xie (2011) find that a recommendation from others is more persuasive when people make decisions for a distant vs. near future consumption. Finally, Agerström and Björklund (2009a, b) find that altruistic, moral behaviors are perceived as more important with greater temporal distance from the moral dilemma, while concrete, selfish temptations take on more weight when the temporal distance from the moral dilemma is near. Based on these considerations, we expect that an allocation policy implemented in a psychologically distant context (e.g., distant future or far vicinity) triggers people to become relatively more concerned with collective outcomes compared to when an allocation policy is implemented in a psychologically close context (e.g., near future or close vicinity) where they are more concerned with individual outcomes (Agerström and Björklund 2009a, b; Eyal and Liberman 2010). Hence:

$\mathrm{H}_{1}$ : Consumers' evaluation of a collective outcome allocation policy (i.e., one with a high collective outcome (focal outcome) and a low individual outcome) increases relative to an individual outcome allocation policy (i.e., one with a 
high individual outcome (focal outcome) and a low collective outcome) in spatially and temporally more distant situations.

\subsection{The activation of a high vested interest mindset by recent personal experience}

A context where consumers compare individual vs. collective allocation policy outcomes is one that may potentially activate a high vested interest mindset, since the outcome can be of high relevance to the consumer and perceived stakes can be large (Crano 1995; Thornton and Knox 2002). According to vested interest theory, contextual manipulations of persuasion processes become less influential, and attitude-behavior correspondence often increases when vested interest is high (Crano 1995; Crano and Prislin 1995; Lehman and Crano 2002). We propose that an individual's recent personal experience with a scarce service resource can trigger the activation of a high vested interest mindset by increasing a consumer's ability to assess the pros and cons of a particular behavior (stake), a consumer's likelihood to be aware of the effects of an action upon oneself (salience) and a consumer's assessment of the chances that a specific event will take place (certainty) (Crano 1995; Morwitz 1997).

The activation of such a high vested interest (internal) mindset is expected to overrule a CLT-based congruency (situationally induced) mindset because consumers with high vested interest are more likely to expend cognitive effort and produce issuerelevant thoughts (Crano 1995; Crano and Prislin 1995; Lehman and Crano 2002). Compared to consumers without recent experience, we expect consumers with recent experience to pay less or no attention to heuristic cues such as the value of fit and the resulting fluency and ease-of-comprehension triggered by the congruency between the allocation policy's focal outcome construal, and spatial and temporal distance. In contrast, these consumers are more likely to use their attitudes as a basis for their decision (cf. striving for an attitude-behavior consistency when vested interest is high; Crano 1995; Crano and Prislin 1995; Lehman and Crano 2002) and to activate concrete, egocentric ("not in my backyard") thoughts. Such thoughts tend to be more tangible and directly experienced (cf. Zhang and Wang 2009) and increase the focus on and evaluation of the individual (relative to the collective) outcome policy, regardless of the temporal or spatial distance. Therefore, we expect that, for allocation policies where individual and collective outcomes are traded off as specified in $\mathrm{H}_{1}$, CLT-based congruency expectations will be overruled for consumers with recent experience who will attach more importance to individual outcomes, irrespective of the temporal and spatial distance present in the policy's decision context. This is related to research of Kim et al. (2009) who show that CLT-based congruency effects hold for novices, but not for experts in the evaluation of political candidates in near vs. distant future situations. Hence:

$\mathrm{H}_{2}$ : Recent experience overrules temporal and spatial distance based congruency effects and increases consumers' evaluation of an individual outcome allocation policy (i.e., one with a high individual outcome (focal outcome) and a low collective outcome) relative to a collective outcome allocation policy (i.e., one with a high collective outcome (focal outcome) and a low individual outcome), irrespective of the spatial and temporal distance. 


\section{Experiment}

\subsection{Method}

Domain To test our expectations, we use a hypothetical experiment in the domain of health care service resources. This domain is a very important economic sector where allocation decisions tend to have a strong societal impact (Reinhardt et al. 2004). Furthermore, it is a sector in which increasingly new allocation policies are introduced (e.g., market-based priority access pricing) that may have large ramifications for consumers. For example, a hospital in the Netherlands recently offered an allocation policy that combined medical urgency criteria with additional access options that offered employers the possibility to pay an extra fee for priority care for their employees (Brouwer and Schut 1999). It is also a very suitable domain to investigate the CLT-based congruency effect as well as the overruling effect of the activation of a high vested interest mindset by recent personal experience. First, health care allocation policies may have strong implications for different outcome types that determine a consumer's mental representation of the policy's focal outcome. In this experiment, we distinguish between allocation policies that either have the collective or individual outcome as focal outcome (referred to as collective outcome policy and individual outcome policy respectively). Second, they are introduced in situational contexts with different psychological distances (e.g., distant future - time gap between proposal and actual launch vs. close future-launch that follows announcement immediately, or far vicinity - service venue located far away vs. close vicinity - close-by service venue). Third, they are evaluated by consumers with different levels of recent personal experience with the health care service provider that may result in different degrees of vested interest mindset activation.

Design The experiment had a 2 (individual vs. collective outcome policy) by 2 (close vs. far psychological distance) by 2 (recent vs. no recent hospital experience) between-subjects factorial design to test whether people evaluate an allocation policy with the collective outcome as focal outcome more (less) favorably in a psychological distant (close) situation than an allocation policy with the individual outcome as focal outcome. To manipulate the focal outcome of the allocation policy, we used the concept of quality of adjusted life years (QALYs) which is the product of gains in quality and length of life as a measure to express the total health improvement resulting from an allocation policy for the participants (Wagstaff 1991). We described an allocation policy with the collective outcome as focal outcome as one where the total number of QALYs saved in society is relatively high (while the total number of QALYs saved for one's own is relatively low). In contrast, an allocation policy with the individual outcome as focal outcome is one where the total number of QALYs saved for one's own is relatively high (while the total number of QALYs saved in society is relatively low). To manipulate the psychological distance in the decision context, we use a joint spatial and temporal distance manipulation and informed participants that the current allocation system of hospitals [in their neighborhood/in the country] became out of date and that therefore a new policy [is going to be implemented within 1 year/will be implemented in 10 years from now], capturing respectively a close and far psychological distance. Recent vs. no recent hospital 
experience was not manipulated but measured for all participants by asking them whether they or a close family member had been to a hospital for treatment in the past 5 years. Based on a pilot test in an earlier study and cut-offs used in practice by institutions within the medical field, we opted for a maximum recent experience level of 5 years (or less) as this was found to be a suitable period to classify participants in terms of their evaluation behavior.

Participants A total of 713 panel members of a Dutch research company, representative for the Dutch population in terms of gender and age, were asked to participate in the study. Thirty-five participants were eliminated because they completed the task in an unusually low or high amount of time, resulting in a total of 678 participants that were used for data analyses. A total of 606 participants had recent hospital experience (for their own treatment or that of a close family member) and 72 participants had no recent hospital experience. The average age of the participants was 47.54 years within a range of $18-83$ years and $47.6 \%$ were males.

Procedure Participants received an email invitation to fill out an online questionnaire about the evaluation of a new allocation policy in the health care sector. After reading the introductory text and after providing a clarification of health care terms like QALYs, which was required knowledge to successfully participate in the questionnaire, participants were randomly assigned to the psychologically close or distant decision context and were asked to evaluate an individual or collective outcome allocation policy. Next, participants received questions related to the perceived closeness of the allocation policy implementation and the construal level of the policy's individual and collective outcomes, and were asked whether they or a close family member had been to a hospital for treatment in the past 5 years to capture recent hospital experience. Finally, respondents were asked to answer some additional questions (e.g., demographics, etc.), and thanked for their participation. The survey took on average about $6.5 \mathrm{~min}$ to complete.

Measures To measure the main dependent variable, attitude toward the allocation policy, participants answered an adapted version of the validated five-item sevenpoint semantic differential scale of Dabholkar (1996) (very bad/very good; very unpleasant/very pleasant; very harmful/very beneficial; very unfavorable/very favorable; and very unsatisfactory/very satisfactory). The five items loaded on the same factor in an exploratory factor analysis (eigenvalue $=4.059,81 \%$ of variance explained, loadings ranging from 0.87 to 0.93 , Cronbach's $\alpha=0.94$ ). To measure the construal level of the allocation policy outcomes, participants received five sevenpoint Likert scale questions for the collective and individual outcome, and were asked to assess whether it was a/an (a) primary, (b) essential, (c) critical, (d) central, or (e) goal-relevant attribute for the evaluation of a new allocation policy. The measures were adapted from Fujita et al. (2006), and Kim et al. (2008) and were reliable (Cronbach's $\alpha=0.95$ and 0.96 for the collective vs. individual outcome, respectively). Finally, to check the effectiveness of the psychological distance manipulation, participants were asked to indicate on a seven-point semantic differential scale (very close/very far) how close they perceived the implementation of the new allocation policy. 


\section{Results}

An independent sample $t$ test revealed that the psychological distance manipulation was perceived as intended $\left(\mu_{\mathrm{CLOSE}}=4.13\right.$ vs. $\left.\mu_{\mathrm{FAR}}=4.63, t(676)=3.907, p<0.001\right)$ and a paired sample $t$ test indicated that, as expected, participants perceived the total number of QALYs saved in society (collective outcome) as more abstract than the total number of QALYs saved for a participant individually (individual outcome) $\left(\mu_{\text {COLLECTIVE }}=4.33\right.$ vs. $\left.\mu_{\text {INDIVIDUAL }}=4.13 ; t(677)=3.11, p<0.01\right)$.

Next, we conducted an ANOVA, with attitude toward the allocation policy as dependent variable, and policy type (individual vs. collective outcome policy), psychological distance (close vs. distant psychological distance), hospital experience (recent vs. no recent experience), and all the two- and three-way interactions as independent variables. We found a main effect of policy type $(F(1,670)=6.52, p<0.05)$, as well as a main effect of hospital experience $(F(1,670)=3.96, p<0.05)$, pointing out that respondents evaluate the individual outcome policy more positively than the collective outcome policy $\left(\mu_{\text {INDIVIDUAL }}=3.85\right.$ vs. $\left.\mu_{\text {COLLECTIVE }}=3.62\right)$, and that those with recent experience have more positive allocation policy evaluations than those without recent experience $\left(\mu_{\text {RECENT EXP }}=3.76\right.$ vs. $\left.\mu_{\text {NO RECENT EXP }}=3.48\right)$. No significant effects emerge for any of the other main and two-way interaction effects, except for the three-way interaction between psychological distance, policy type, and hospital experience $(F(1,670)=4.07, p<0.05)$. Figure 1 shows this significant three-way interaction effect in a graphical way.

When we look at the relevant simple effects in the two hospital experience conditions, we can further interpret these findings. For participants without recent hospital experience (panel a: no activation of high vested interest mindset), we find support for the predicted interaction of psychological distance and allocation policy type $(F(1,68)=4.94, p<0.05)$. In line with the CLT-based congruency expectation $\left(\mathrm{H}_{1}\right)$, the individual outcome allocation policy is evaluated more positively than the collective outcome allocation policy in the spatially and temporally close (vs. distant) situation. In contrast, for participants with recent hospital experience (panel b: activation of high vested interest mindset), the individual outcome policy is evaluated more positively in both the spatially and temporally close and distant situation with a significant main effect for policy type $(F(1,602)=6.47, p<0.05)$ and no significant effects for the other main and two-way interaction effects. This further confirms the overruling effect of the activation of a high vested interested mindset by a consumer's recent experience with the service resource provider. Hence, in summary we find support for $\mathrm{H}_{2}$ that recent personal experience activates a high vested interest mindset that overrules the CLT-based congruency effect (through the significant three-way interaction), and we can only confirm $\mathrm{H}_{1}$ for participants without recent hospital experience where there is no activation of a high vested interest mindset.

\section{General discussion}

We find that collective outcomes are perceived as more abstract than individual outcomes, and that the evaluation of a health care service allocation policy with a focus on the individual outcome relative to that with a focus on the collective 
Fig. 1 a No recent hospital experience (no activation of high vested interest mindset). b Recent hospital experience (activation of high vested interest mindset)

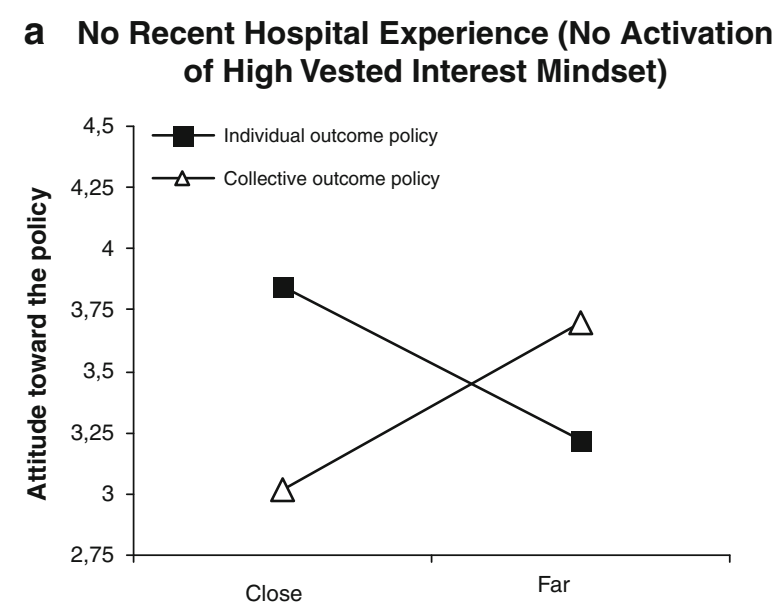

Spatial and temporal distance

b Recent Hospital Experience (Activation of High Vested Interest Mindset)

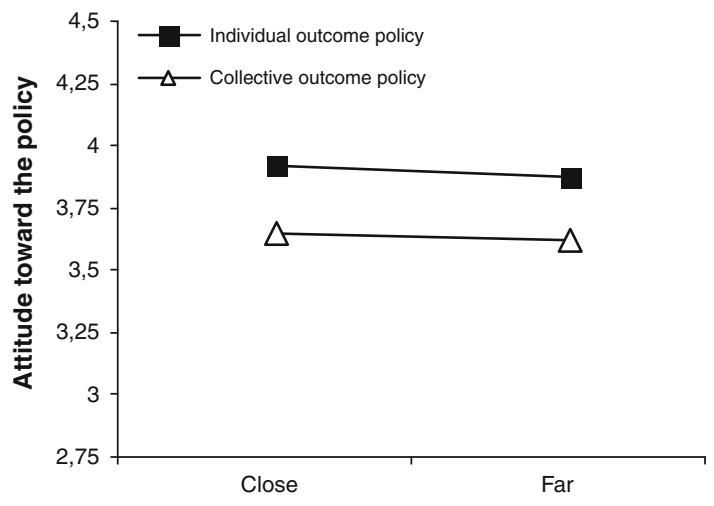

Spatial and temporal distance

outcome increases (decreases) in a spatially and temporally close (distant) decision context. This confirmation of the CLT-based congruency expectation is only supported for consumers that did not have recent hospital experience (Fig. 1a). For those that did have recent hospital experience, we find that the individual outcome allocation policy is evaluated more positively than the collective outcome allocation policy in both a close as well as a far spatially and temporally distant situation (Fig. 1b). We therefore find support for the proposition that recent personal experience tends to activate a high vested interest internal mindset that overrules a situationally induced mindset triggered by the temporal and spatial distance of the decision context.

Our finding is in line with vested interest theory that shows that, when vested interest is high, contextual manipulations of persuasion processes - such as congruency effects - often become less influential, and attitude-behavior correspondence increases (Crano 1995; Crano and Prislin 1995; Lehman and Crano 2002). But, we 
also show that, within a context where individual and collective outcomes are traded off (a potentially high vested interest context), vested interest is only activated for consumers that have recent experience. Vested interest theory indeed confirms that vested interest is strengthened whenever there is more at stake (stake), whenever the action is more prominent (salience) and more likely to occur (certainty) (Crano 1995; Crano and Prislin 1995; Lehman and Crano 2002). We therefore provide evidence that recent experience is likely to strengthen vested interest by increasing each of the three sub-components of stake, salience, and certainty. This high vested interest mindset stimulates the activation of an (internal) concrete, egocentric ("not in my backyard") mindset that trumps the CLT-based congruency effect of the (situational) mindset triggered by the spatial and temporal distance of the decision context. This is in line with Zhang and Wang (2009) who show that distance dimensions that are more tangible and directly experienced (in our case the internal mindset) will have a stronger effect on other dimensions (in our case overrule the situationally induced mindset).

\subsection{Academic contributions, limitations, and future research}

Applying CLT in the context of health care allocation policy implementations is important because it focuses on a relatively unexplored field of research that is of paramount importance for society. Trope (2004), for instance, observed that, although the societal implications of CLT are substantial, CLT has not often been tested with stimuli that have clear implications for society. In addition, regularly new health care allocation policies are developed that may differ on the abstractness of the allocation policy outcome type and that may be implemented in decision contexts with different psychological distances, making the CLT literature particularly suitable for this important domain.

The main contribution of this study is that we demonstrate and explain why allocation policy evaluations differ from what would be expected from CLT due to the activation of consumers' vested interest (i.e., when consumers have recent experience with the service provider). More specifically, the finding that the effect of the mindset triggered by the spatial and temporal distance of the decision context (i.e., the CLT-based expectation) is overruled by the activation of a vested interest internal mindset triggered by recent personal experience is a novel finding in the CLT literature. Our results relate to the finding of Kim et al. (2009) who show that expertise moderates the evaluation of political candidates over temporal distance, with CLT-based congruency effects holding for novices, but not for experts. We add to this finding by showing that an internal mindset may also overrule (rather than simply nullify) a situationally induced mindset. In cases where a high vested interest mindset is activated, which was the case for participants with recent hospital experience in a context where individual outcomes were at stake, consumers more likely use their attitudes as a basis for their decision, activate concrete, egocentric ("not in my backyard") thoughts and put less or no attention on contextual manipulations. As a result, these consumers evaluate an allocation policy that focuses on individual outcomes higher than a policy that focuses on collective outcomes irrespective of the spatial and temporal distance in the decision context. To the best of our knowledge, we are the first to show such overruling effects of the activation of a vested interest 
mindset by recent personal experience on the previously found CLT-based congruency effect.

Some limitations of our study are worth noting that also open up avenues for possible further research. First, our respondents were shown a hypothetical scenario which did not have actual consequences for them. Although we did find important differences for respondents with and without recent hospital experience, it would be interesting to investigate allocation policy evaluations for real allocation policy implementations. Second, respondents were asked to evaluate allocation policy implementations in a health care setting. Although we believe that our results are relevant for a wide range of contexts, it would be interesting to extend our research for other domains that are confronted with a scarcity of service resources which is often the case in recreation and education industries. Finally, we measured recent experience using a threshold of 5 years to determine whether someone (oneself or a close family member) has or has no recent hospital experience. It would be interesting to use other thresholds that may result in a more balanced sample size of consumers with vs. without recent experience and/or to include experience as a continuous (instead of discrete) variable in the analyses.

\subsection{Managerial implications}

Recognizing that individuals weigh collective vs. individual outcomes differently depending on the distance present in the decision context has important ramifications for society and social welfare. Our research shows that allocation policies that focus on individual outcomes benefit most when they are introduced in a close psychological distance context, e.g., with a less distant temporal timing and/or for a less remote location. Depending on the launch time of the allocation policy and the service provider's location, managers and policy makers should (or should not) highlight the temporal or spatial distance. If the temporal or spatial frame is incongruent with the mental representation of the allocation policy's focal outcome, they could use other distance manipulations, such as using a more vs. less hypothetical situation (e.g., announcing the policy with an imaginary vs. real example) and/or focusing on more vs. less socially distant others (e.g., announcing the policy with an example that describes strangers vs. friends).

It is also important for decision makers like hospital managers to realize that the activation of a high vested interest mindset triggered by consumers' recent experiences with the resource provider may overrule a situationally induced mindset triggered by the temporal and spatial distance in the decision context. From the moment a consumer has a high vested interest in the allocation policy outcome because $\mathrm{s} /$ he has recent personal experience, it is crucial for decision makers to realize that consumers are highly involved and tend to generate concrete, egocentric thoughts as a basis for their decision, thereby paying less or no attention to contextual manipulations. Since these consumers prioritize individual outcomes, policy makers should highlight the individual benefits (or minimize the loss of them), using strong arguments. In contrast, peripheral cues that maximize the value of fit triggered by the CLT-based congruency effect do work in cases where the consumer has no recent personal experience.

Psychological distance and a consumer's internal mindset are thus important elements that need to be considered by decision makers, like hospital managers, 
and by extension other allocation policy decision makers, in their decisions on when and how to communicate about the allocation policy's decision announcement. Using these insights can help to increase the evaluation of allocation policies for scarce resources. Decision makers may benefit from this because it will result in higher public support and thus an easier policy implementation process (Weiss 1999).

Acknowledgments The authors thank the editor and two anonymous reviewers for the valuable suggestions in the review process. They also thank Dirk Smeesters and Wouter Vanhouche for valuable comments on a previous version of the paper.

Open Access This article is distributed under the terms of the Creative Commons Attribution Noncommercial License which permits any noncommercial use, distribution, and reproduction in any medium, provided the original author(s) and source are credited.

\section{References}

Agerström, J., \& Björklund, F. (2009a). Moral concerns are greater for temporally distant events and are moderated by value strength. Social Cognition, 27(2), 261-282.

Agerström, J., \& Björklund, F. (2009b). Temporal distance and moral concerns: future morally questionable behavior is perceived as more wrong and evokes stronger prosocial intentions. Basic and Applied Social Psychology, 31(1), 49-59.

Brouwer, W. B. F., \& Schut, F. T. (1999). Priority care for employees: a blessing in disguise? Health Economics, 8(1), 65-73.

Crano, W. D. (1995). Attitude strength and vested interest. In R. E. Petty \& J. A. Krosnick (Eds.), Attitude strength: Antecedents and consequences (pp. 131-157). Mahwah: Erlbaum.

Crano, W. D., \& Prislin, R. (1995). Components of vested interest and attitude-behavior consistency. Basic and Applied Social Psychology, 17(1).

Dabholkar, P. A. (1996). Consumer evaluations of new technology-based self-service options: an investigation of alternative models of service quality. International Journal of Research in Marketing, 13(1), 29-51.

Eyal, T., \& Liberman, N. (2010). Morality and psychological distance: a construal level theory perspective. paper presented at the Herzliya Symposium on Personality and Social Psychology.

Fujita, K., Trope, Y., Liberman, N., \& Levin-Sagi, M. (2006). Construal levels and self-control. Journal of Personality and Social Psychology, 90(3), 351-367.

Kahneman, D., Knetsch, J. L., \& Thaler, R. H. (1986). Fairness and the assumptions of economics. Journal of Business, 59(4), 285-300.

Kardes, F. R., Cronley, M. L., \& Kim, J. (2006). Construal-level effects on preference stability, preferencebehavior correspondence, and the suppression of competing brands. Journal of Consumer Psychology, 16(2), 135-144.

Kim, K., Zhang, M., \& Li, X. (2008). Effects of temporal and social distance on consumer evaluations. Journal of Consumer Research, 35(4), 706-713.

Kim, H., Rao, A. R., \& Lee, A. Y. (2009). It's time to vote: the effect of matching message orientation and temporal frame on political persuasion. Journal of Consumer Research, 35(6), 877-889.

Lehman, B. J., \& Crano, W. D. (2002). The pervasive effects of vested interest on attitude-criterion consistency in political judgment. Journal of Experimental Social Psychology, 38(2), 101-112.

Liberman, N., \& Trope, Y. (1998). The role of feasibility and desirability considerations in near and distant future decisions: a test of temporal construal theory. Journal of Personality and Social Psychology, 75 (1), 5-18.

Liberman, N., Trope, Y., \& Wakslak, C. (2007). Construal level theory and consumer behavior. Journal of Consumer Psychology, 17(2), 113-117.

Lindholm, L. A., Emmelin, M. A., \& Rosén, M. E. (1997). Health maximization rejected: the view of Swedish politicians. European Journal of Public Health, 7(4), 405-410.

Liviatan, I., Trope, Y., \& Liberman, N. (2008). Interpersonal similarity as a social distance dimension: implications for perception of others' actions. Journal of Experimental Social Psychology, 44(5), 1256-1269. 
Milkman, K. L., Rogers, T., \& Bazerman, M. H. (2010). I'll have the ice cream soon and the vegetables later: a study of online grocery purchases and order lead time. Marketing Letters, 21(1), 17-35.

Morwitz, V. G. (1997). Why consumers don't always accurately predict their own future behavior. Marketing Letters, 8(1), 57-70.

Novemsky, N., Dhar, R., Schwarz, N., \& Simonson, I. (2007). Preference fluency in choice. Journal of Marketing Research, 44(3), 347-356.

Persad, G., Wertheimer, A., \& Emanuel, E. J. (2009). Principles for allocation of scarce medical interventions. Lancet, 373(9661), 423-431.

Reinhardt, U. E., Hussey, P. S., \& Anderson, G. F. (2004). U.S. health care spending in an international context. Health Affairs, 23(3), 10-25.

Sanna, L. J., Lundberg, K. B., Parks, C. D., \& Chang, E. C. (2010). Think and act globally, think and act locally: cooperation depends on matching construal to action levels in social dilemmas. Journal of Experimental Social Psychology, 46(6), 1126-1129.

Soman, D., Ainslie, G., Frederick, S., Li, X., Lynch, J., Moreau, P., et al. (2005). The psychology of intertemporal discounting: why are distant events valued differently from proximal ones? Marketing Letters, 16(3/4), 347-360.

Thornton, B., \& Knox, D. (2002). "Not in my back yard": the situational and personality determinants of oppositional behavior. Journal of Applied Social Psychology, 32(12), 2554-2574.

Trope, Y. (2004). Theory in social psychology: seeing the forest and the trees. Personality and Social Psychology Review, 8(2), 193-200.

Trope, Y., \& Liberman, N. (2003). Temporal construal. Psychological Review, 110(3), 403-421.

Trope, Y., \& Liberman, N. (2010). Construal-level theory of psychological distance. Psychological Review, $117(2), 440-463$.

Trope, Y., Liberman, N., \& Wakslak, C. (2007). Construal levels and psychological distance: effects on representation, prediction, evaluation, and behavior. Journal of Consumer Psychology, 17(2), 83-95.

Wagstaff, A. (1991). QALYs and the equity-efficiency trade-off. Journal of Health Economics, 10(1), 2141.

Weiss, C. H. (1999). The interface between evaluation and public policy. Evaluation, 5(4), 468-486.

White, K., Macdonnel, R., \& Dahl, D. W. (2011). It's the mindset that matters: the role of construal level and message framing in influencing consumer efficacy and conservation behaviors. Journal of Marketing Research, 48(3), 472-485.

Zhang, M., \& Wang, J. (2009). Psychological distance asymmetry: the spatial dimension vs. other dimensions. Journal of Consumer Psychology, 19(3), 497-507.

Zhao, M., \& Xie, J. (2011). Effects of social and temporal distance on consumers' responses to peer recommendations. Journal of Marketing Research, 48(3), 486-496. 